\title{
NON-BPS BLACK HOLES AND SELF-INTERACTING FUNDAMENTAL STRINGS
}

\author{
DIEGO VALERIO CHIALVA \\ Nordita Institute, Roslagstullsbaken 23, 10691 Stockholm, Sweden. E-mail: chialva@nordita.org .
}

\begin{abstract}
The string-black hole correspondence principle can be investigated in the non-BPS scenario by studying the string configuration and entropy when the string coupling is slowly increased. Through a rigorous analysis, it is shown how an ensemble of string states at fixed mass and Neveu-Schwarz charges gets dominated in any dimension by compact states for which the one-loop corrections are important (possibly signaling the transition to a black hole regime/description) and with a size (spread) within the horizon radius of the expected correspondent black holes.
\end{abstract}

The relation between the Hawking-Bekenstein entropy of black holes and the counting of microstates is one of the most studied topics in black holes physics. In this short communication we deal with the non-BPS scenario in the setup of closed superstring theory. In doing so, we touch upon some important questions: how to individuate the relevant microstates, how and when the two descriptions (general relativity and quantum string) match and, finally, how geometric features of black holes arise from the quantum description.

Within string theory, the string-black hole correspondence principle ${ }^{1}$ states that a classical black hole is in one-to-one correspondence with an ensemble of string and/or D-brane states (depending on its type of charges).

Two quantities in particular play a key role in the analysis: the string coupling $g_{s}$ ब and the black hole horizon radius $R_{B H}$, especially in relation with the string length scaleb $l_{s}=\sqrt{\alpha^{\prime}}$.

The string and the black hole entropy are conjectured to become equal at a correspondence point $g_{s} \sim\left(M^{2}-Q^{2}\right)^{-\frac{1}{4}} \equiv g_{c}$ in parameter space, where $M$ is the mass of the string states in the ensemble, equal to the black hole's one at this point, and $Q^{i}$ are the Neveu-Schwarz charge $\mathrm{C}$. Also, at this point $R_{B H}$ approaches $l_{s}$.

There are major obstacles to verifying such a statement within string theory. We must show what happens to the relevant ensemble of string states and their entropyd when the string coupling (adiabatically) increases巴 from $g_{s}=0$ to $g_{s}=g_{c}$.

a Linked to the Newton's constant ad the string length at the perturbative level as $G_{N} \sim g_{s}^{2}\left(\alpha^{\prime}\right)^{\frac{d-1}{2}}$ in $d=D-1$ extended spatial dimensions.

${ }^{\mathrm{b}}$ In what follows the horizon radius is to be intended in the string frame. The general relativity description is valid when the curvature at $R_{B H}$ (in the string frame) is smaller than the string scale, this yields the condition $R_{B H}>l_{s}$.

${ }^{\mathrm{c}}$ Here, for simplicity $\left|Q_{L}^{i}\right|=\left|Q_{R}^{i}\right|=\left|Q^{i}\right|, Q^{2}=\sum\left(Q^{i}\right)^{2}$, where $L(R)$ refer to the (anti)holomorphic part of the string.

${ }^{d}$ We will consider single-string entropy, which dominates the contribution to the total entropy.

eThis is what makes the comparison safest in the BPS case, due to the non-renormalization properties of BPS state counting. In the non-BPS case, instead, one has to compute the corrections to the microstate counting when interactions are turned on. 
This entails coping with two main issues: i) computing the relevant corrections to the tree-level results due to interactions, ii) correctly defining and measuring the size of string states and their size distribution and check its relation with the horizon radius of the expected correspondent black hole.

Refs. 2[3 investigated these issues within string theory. In fact, these problems where previously treated in approximated models, leading to a variety of results $\frac{4}{4}$ but never in fully rigorous string formalism.

In coping with ii), the traditional approach of defining a size operator for the string failed to comply with the requirements of the theory. Ref. 2, instead, measured the size of an object through a well-defined operational procedure. The basic idea is that, in a theory where the observables are scattering amplitudes, the size of an object is measured by scattering probesf over it. For mixed states (ensembles), the form factor obtained in this way can be related to their size distribution.

In a microcanonical ensembleg, the entropy of the superstring at zero coupling and fixed (tree) mass level $M_{0}^{2}=Q_{L(R)}^{2}+N_{L(R)}$, Neveu-Schwarz charges $Q_{L(R)}^{i}$ and size $R$ was found to be $\left(\alpha^{\prime}=4, \mathcal{N}=\sqrt{N_{L}}+\sqrt{N_{R}}\right)$

$$
S=\pi \mathcal{N} \sqrt{d-1}-\frac{3 \sqrt{d-1}}{8 \mathcal{N} \pi} R^{2}+\ln \left(\frac{R^{d-1}}{N_{L}^{\frac{d+2}{4}} N_{R}^{\frac{d+2}{4}} \mathcal{N}^{\frac{d}{2}}}\right)
$$

Turning on interactions $\left(g_{s} \neq 0\right)$ yields corrections to the free-string result. Neglecting those related to $R$ (renormalization of the size), the relevant ones come from the string mass-shift.

The study of mass shifts in string theory is very difficult because very few physical vertex operators for massive string states are actually known (in covariant gauge) and also because of the intrinsic complications in the computations of amplitudes. ${ }^{5}$

In order to obtain the average squared mass shift for the relevant ensemble of states at fixed tree-level mass charges and size, Ref. 3 exploited two key properties of the string amplitudes. First, the unitarity of the string S-matrix and, then, the modular and periodicity properties of the string amplitudes on the torus, which allowed to determine a system of recurrent equations for the amplitude itsel .

Eventually, the average squared mass shift for the ensemble of string states at fixed mass and chargesi was found $\mathrm{d}^{3}$ to $b e^{\mathrm{j}}$

$$
{\overline{\Delta M^{2}}}_{\left.\right|_{N, Q}}=-g_{s}^{2}\left(M^{2}-Q^{2}\right)^{1+\frac{3-D}{4}},
$$

\footnotetext{
${ }_{\mathrm{f}}^{\mathrm{f}}$ In our case, a linear combination of graviton, dilaton and Kalb-Ramond field as we are interested in the mass distribution of the object.

${ }^{g}$ Which appears to be the only well-defined one for closed string states due to the level matching conditions.

${ }^{\mathrm{h}}$ Namely for its coefficients, once the amplitude is expanded in powers of suitable variables, see Ref. 3

${ }^{\mathrm{i}}$ Again, we consider for simplicity $\left|Q_{L}^{i}\right|=\left|Q_{R}^{i}\right|=\left|Q^{i}\right|, N_{L}=N_{R}=N$.

$\mathrm{j}$ This formula tells us that the interaction in the effective Lagrangian will become important - of order one - at $g_{s e} \sim\left(M^{2}-Q^{2}\right)^{\frac{d-6}{8}}$ as expected from field theory considerations. We are writing the result in terms of the physical mass $M$, which is probably an even more accurate estimate.
} 
while the average squared mass shift for the ensemble of states at fixed $M, Q^{i}, R$ is 2

$$
{\overline{\Delta M^{2}}}_{\left.\right|_{M, Q, R}}=-g_{s}^{2}\left(M^{2}-Q^{2}\right) R^{2-d},
$$

absorbing an inessential proportionality constant in $g_{s}$. We can then infer the corrections to the single-string entropy $S$ when the $g_{s}$ is adiabatically increased ${ }^{2}$

$$
e^{S} \sim e^{2 \pi \sqrt{d-1}\left(\sqrt{M^{2}-Q^{2}}+g_{s}^{2} \frac{\sqrt{M^{2}-Q^{2}}}{2 R^{d-2}}-\frac{3}{32 \pi^{2} \sqrt{M^{2}-Q^{2}}} R^{2}\right)}
$$

In particular, we see that for $2^{-1} g_{s}^{2} \sqrt{M^{2}-Q^{2}} R^{2-d} \geq 1$, the entropy becomes dominated by strings whose size is $R \lesssim R_{b} \sim\left(g_{s}^{2} \sqrt{M^{2}-Q^{2}}\right)^{\frac{1}{d-2}}$, which has the same perturbative formula of the horizon radius of the correspondent black hole. It approaches the string length at $g_{s} \sim\left(M^{2}-Q^{2}\right)^{-\frac{1}{4}}$, in accordance with the correspondence principle.

The string entropy is dominated in particular by the states with the minimum possible size. It can be shown ${ }^{2}$ that perturbation theory is sufficient to determine the minimal size only in $d=3$, yielding $R_{\min }=\frac{2}{g_{s}^{2}\left(M^{2}-Q^{2}\right)}$. It is finally interesting to observe that the relative average one-loop mass shift for these minimum size states is of order $\frac{\left.\overline{\Delta M^{2}}\right|_{M, Q}, R_{\min }}{M^{2}} \sim O(1)$, possibly signaling a radical change in the relation between entropy and mass for $g_{s}>g_{c}$ and hinting to a transition between a perturbative string regime and a black hole one. Finally, the corrected formula for the entropy at leading order in $d=3$ is 2

$$
S(M, Q) \sim 2 \pi \sqrt{2} \sqrt{M^{2}-Q^{2}}\left(1+\frac{g_{s}^{4}\left(M^{2}-Q^{2}\right)}{4}\right) .
$$

\section{Acknowledgments}

I would like to thank very much Gabriele Veneziano and Paolo di Vecchia for the many illuminating conversations about this subject of research.

\section{References}

1. L. Susskind, In *Teitelboim, C. (ed.): The black hole* 118-131. arXiv:hep-th/9309145 E. Halyo, A. Rajaraman and L. Susskind, Phys. Lett. B 392 (1997) 319 arXiv:hep-th/9605112. E. Halyo, B. Kol, A. Rajaraman and L. Susskind, Phys. Lett. B 401 (1997) 15 arXiv:hep-th/9609075]. G. T. Horowitz and J. Polchinski, Phys. Rev. D 55 (1997) 6189 arXiv:hep-th/9612146]. L. Susskind, Phys. Rev. Lett. 71 (1993) 2367 arXiv:hep-th/9307168].

2. D. Chialva, Nucl. Phys. B 819 (2009) 256 arXiv:0903.3977 [hep-th]].

3. D. Chialva, Nucl. Phys. B 819 (2009) 225 arXiv:0903.3979 [hep-th]].

4. G. T. Horowitz and J. Polchinski, Phys. Rev. D 57 (1998) 2557 arXiv:hep-th/9707170. T. Damour and G. Veneziano, Nucl. Phys. B 568 (2000) 93 arXiv:hep-th/9907030.

5. N. Seiberg, Phys. Lett. B 187 (1987) 56. A. Sen, Nucl. Phys. B 304 (1988) 403. J. Polchinski, Nucl. Phys. B 307 (1988) 61.

${ }^{\mathrm{k}}$ In the Einstein frame, which must be used to compute the black hole entropy. 